\title{
Anxiety, depression, physical symptoms, and activity in patients with hematological malignancy undergoing chemotherapy: A cross-sectional study
} \author{
Kenjiro Mori' ${ }^{6}$ and Yoko Kusuba ${ }^{7}$ \\ ${ }^{1}$ Institute of Biomedical Sciences (Health Sciences), Nagasaki University, Nagasaki, Japan \\ ${ }^{2}$ Department of Oncology and Rehabilitatoion, National Cancer Center Hospital, Tokyo, Japan \\ ${ }^{3}$ Graduate School of Health Sciences, Gunma University, Gunma, Japan \\ ${ }^{4}$ Department of Rehabilitation, Michinoo-Miyata Orthopedic Clinic, Nagasaki, Japan \\ ${ }^{5}$ Department of Rehabilitation, Nagasaki University Hospital, Nagasaki, Japan \\ ${ }^{6}$ Department of Rehabilitation, Nagasaki KITA Hospital, Nagasaki, Japan \\ ${ }^{7}$ School of Nursing at Fukuoka, International University of Health and Welfare, Fukuoka, Japan
}

Jiro Nakano"*, Takuya Fukushima ${ }^{2}$, Koji Tanaka ${ }^{3}$, Shun Ishii ${ }^{4}$, Ayumi Natsuzako ${ }^{5}$, Kazumi Ueno ${ }^{1}$, Emi Matsuura ${ }^{1}$, Kaori Hashizume ${ }^{1}$,

\begin{abstract}
Objective: The purpose of this study was to investigate the prevalence and characteristics of depression and anxiety in patients with hematological malignancy including examining their relationship with physical functioning and physical symptoms.

Method: Japanese inpatients ( $\mathrm{n}=173$, mean age: $62.7 \pm 15.0$ years) with hematological malignancy undergoing chemotherapy and 115 age- and sex-matched healthy controls participated. All participants completed the Hospital Anxiety and Depression Scale. For patients with hematological malignancy, muscle strength, physical activity, activities of daily living, and quality of life (European Organisation for Research and Treatment of Cancer Quality of Life Questionnaire-C30) were assessed.

Results: We revealed that $32.9 \%$ and $45.7 \%$ of patients had anxiety and depression, respectively, which were both significantly higher than the values among healthy controls. Patients with hematological malignancy who had anxiety and depression displayed lower physical functioning scores and higher symptom scores on the QLQ-C30. Logistic regression analyses revealed that age, nausea, and cognitive functioning were significantly associated with anxiety in patients with hematological malignancy, and insomnia was significantly associated with depression.

Significance of Discussion: The prevalence of anxiety and depression was high in Japanese inpatients with hematological malignancy undergoing chemotherapy. Anxiety and depression may be affected more by physical symptoms than by physical functioning or activity; therefore, the relationship between psychological distress (anxiety and depression) and physical symptoms should be focused on when treating patients with hematological malignancy undergoing chemotherapy.
\end{abstract}

\section{Introduction}

Patients with hematological malignancy present with a variety of physical and mental symptoms such as cytopenia derived from nutritional deficiencies and bone marrow suppression [1], nausea/ vomiting [2], anorexia [3], and cancer-related fatigue [4]. In addition, among these patients, anxiety and depression can potentially impair their physical and social functioning and quality of life (QoL) $[5,6]$. However, the severity of anxiety and depression among patients with hematological malignancy has not been compared with that of healthy people in previous studies.

Notably, a few reports compared anxiety and depression between patients with other cancers and healthy people. Concerning breast cancer, some reports revealed that anxiety and depression did not differ between patients and healthy controls [7-9]. Further, it was reported that anxiety and depression among patients with lung cancer was severer than that of healthy people who lived in suburban areas, but not urban areas [10]. Severer depression and anxiety were also observed in inpatients with colorectal and testicular cancer as compared to healthy people [11,12].
Key factors associated with anxiety and depression have been addressed in several reports examining patients with cancer; however, some did not compare against healthy people. For example, in a study about inpatients with hematological malignancy in Saudi Arabia, the associated factors of anxiety and depression were multiple comorbidities and a tense home atmosphere, but not age, income, or treatment [13]. Further, research in China revealed that optimism and resilience, but not self-efficacy, were associated with depression and anxiety among inpatients with hematological malignancy [14]. In outpatients, depression and anxiety are aggravated by smoking and having to relocate for treatment [15]. Moreover, in a study that investigated QoL

${ }^{\star}$ Correspondence to: Jiro Nakano, Institute of Biomedical Sciences (Health Sciences), Nagasaki University, 1-7-1 Sakamoto, Nagasaki-shi, Nagasaki 8528520, Japan, Tel: +81-95-819-7919; E-mail: nakano-j@nagasaki-u.ac.jp

Key words: hematological malignancy, anxiety, depression, physical function, physical symptom

Received: June 15, 2019; Accepted: July 01, 2019; Published: July 10, 2019 
and physical symptoms, social functioning, nausea, insomnia, and financial difficulties were all associated with depression and anxiety among patients with hematological malignancy [16]. Lastly, fatigue and sex also influenced mental distress in patients with hematological malignancy [4,17].

Consequently, various factors are associated with depression and anxiety; however, the affecting factors in patients with hematological malignancy have not been fully clarified. Especially, few studies have demonstrated the relationship between depression and anxiety and physical functioning such as muscle strength, activities of daily living (ADL), and physical activity in patients with hematological malignancy. It has been posited that physical functioning of patients with hematological malignancy decreases during hospital treatment (e.g., receiving chemotherapy) [18]. There is also a high possibility that anxiety and depression are affected by physical functioning [19].

Therefore, we investigated the prevalence and characteristics of anxiety and depression in Japanese inpatients with hematological malignancy undergoing chemotherapy including examining their relationship with physical functioning and physical symptoms. Moreover, we explored the risk factors of anxiety and depression.

\section{Methods}

\section{Study design and participants}

This cross-sectional observational study was conducted at Nagasaki University Hospital, from January 2014 to March 2019. Inpatients with hematological malignancy undergoing chemotherapy in Nagasaki University Hospital and age- and sex-matched healthy controls in Nagasaki city participated. Informed consent was obtained from all participants. Inclusion criteria of patients included the following: 1) inpatients diagnosed with hematological malignancy including lymphoma, acute myeloid leukemia and Acute lymphoblastic leukemia, 2) hospitalized to receive chemotherapy, and 3) aged $>20$ years. Exclusion criteria included the following: 1) difficulty communicating, 2) critical condition, 3) had received radiotherapy or transplant treatment, 4) severe heart disease, 5) severe respiratory disease, 6) hepatic or renal failure, or 7) cerebrovascular or orthopedic disease that greatly influenced physical functioning. Healthy controls were recruited at health-related events and welfare businesses for citizens.

General information about participants such as age, sex, height, weight, and body mass index (BMI) were recorded. For patients, clinical information was also collected, including diagnosis; days from hospitalization; disease duration; treatment type; and blood chemistry data such as serum albumin, serum C-reactive protein, and hemoglobin concentration. Anxiety, depression, and muscle strength were also measured for all participants. Additionally, ADL, physical activity, and QoL were assessed for patients.

This study was approved by a hospital ethics committee before study commencement (approval number 15072757). Study objectives were explained to all participants before they were invited to participate. Written consent was obtained from all consenting participants.

\section{Psychological distress}

The Hospital Anxiety and Depression Scale (HADS) was used to assess psychological distress [20]. This is a widely used valid questionnaire to measure psychological morbidity in cancer patients [20]. It consists of a 7-item anxiety subscale and a 7-item depression subscale. Each item is rated on a scale from 0 (best status) to 3 (worst status); possible scores for each subscale range from 0 to 21 . Scores between 0 and 7 suggest "no case," 8 to 10 represents a "possible case," and 11 to 21 indicates a "probable case of anxiety/depression" [21]. In this study, a score of 8 was set as the cut-off value of anxiety/depression per a previous study [21].

\section{Muscle strength}

Handgrip strength was measured as a marker of general muscle strength using a standard adjustable-handle dynamometer (TKK 5101; Takei Scientific Instruments Co. Ltd., Japan). Measurements were taken in standardized position with participants standing with their arm held in zero abduction flush against their body. A single attempt of maximum right- and left-hand grip strength was recorded in kilogram force, and higher values were adopted for analyses.

Additionally, the strength of the quadriceps femoris muscle was assessed. Muscle strength was quantified using maximum voluntary isometric contraction for knee joint extension, per a previous study [22]. Participants were seated at the corner of a bed or chair with their knees at about 90 degrees flexion. A portable muscle force dynamometer ( $\mu$-tas MF-01, ANIMA Corp., Japan) was placed against the anterior side of participants' legs just proximal to the malleoli. The dynamometer stabilizing belt passed around the legs of the bed/chair. Participants were then asked to continue trying to straighten their knee as hard as possible until the tester asked them to stop. The measurement was performed for both the right and left legs, and data from the stronger leg were used for analyses.

\section{Activities of daily living}

In patients with hematological malignancy, ADL was evaluated using the Functional Independence Measure, which assesses physical and cognitive functioning and the need for assistance. It consists of an 18 -item scale that measures independence in performing tasks involved in feeding, grooming, dressing, toileting, mobility, and cognition [23]. Each item is rated on a scale from 1-7 ( $1=$ total assistance, $2=$ maximal assistance, $3=$ moderate assistance, $4=$ minimal assistance, 5 = needs supervision, $6=$ modified independence, and $7=$ Complete independent).

\section{Physical activity}

The Eastern Cooperative Oncology Group performance status was defined as follows: $0=$ fully active and able to perform all pre-disease activities without restriction; 1 = restricted in physically strenuous activity; however, ambulatory and able to perform work of a light or sedentary nature; 2 = ambulatory and capable of all selfcare; however, unable to complete any work activities, and "up and about" for more than $50 \%$ of waking hours; 3 = capable of only limited selfcare and confined to bed or chair for more than $50 \%$ of waking hours; and $4=$ completely disabled, cannot perform any selfcare, and totally confined to a bed or chair.

Daily steps were measured as a marker of physical activity using a pedometer with accelerometer. Participants were instructed to wear the accelerometer (Kenz Lifecorder GS; Suzuken Co., Ltd., Japan) on their belt or waistband, above the right midline of the thigh, from the moment they got out of bed in the morning until they went to bed in the evening, except while bathing. The wearing period was 1 week and mean daily step count was calculated.

\section{Quality of life}

QoL was measured using The European Organisation for Research and Treatment of Cancer QoL Questionnaire-C30 (EORTC QLQ-C30). 
The EORTC QLQ-C30 is a cancer-specific self-report questionnaire and consists of a global QoL scale (two items), functional scales (physical, role, emotional, cognitive, and social functioning), and symptom scales (fatigue, pain and nausea/vomiting, insomnia, dyspnea, appetite loss, diarrhea, constipation, and financial difficulties). The items are scored on a 4-point categorical scale ranging from 1 (not at all) to 4 (very much), except the global QoL scale, which utilizes a modified 7-point linear analogue scale. All scales are transformed to a score from 0-100. For the global QoL scale and functional scales, higher scores represent better functioning. For the symptom scales, higher scores indicate severe symptoms [24].

\section{Data analyses}

Data were analyzed using SPSS 22.0 (IBM, NY, USA). Data were shown as means \pm standard deviation for continuous data and as frequencies (\%) for categorical data. First, patients were compared with healthy controls regarding anxiety and depression. Second, patients were divided into two groups based on their HADS anxiety/depression subscale scores ( $\geq 8=$ anxious/depressed group and $<8=$ non-anxious/ depression group). Variables between groups were compared using independent samples t-tests and $\chi^{2}$ tests. Third, all variables with a significant association with anxiety/depression were entered into a multiple stepwise logistic regression model, with the dichotomous anxiety/depression as the dependent variable, to explore psychological status factors. $\mathrm{P}<.05$ was considered statistically significant.

\section{Results}

One-hundred seventy-three patients with hematological malignancy being treated at Nagasaki University Hospital were recruited, and data were collected from 115 healthy controls. There were no differences between patients and controls regarding general characteristics (Table 1).

Anxiety and depression in patients with hematological malignancy increased compared with healthy controls. Patients had significantly higher mean HADS-anxiety and HADS-depression scores than did controls; however, when the presence of anxiety was judged using the cut-off value (HADS-anxiety $>8$ points), the prevalence of anxiety in patients and healthy controls was not significantly different. Further, regarding muscle strength, both handgrip and knee extension strength significantly decreased in patients compared with controls (Table 1).

Patients' general and clinical characteristics are shown in Table 2. Most cancer type was lymphoma (45.7\%), followed by acute myeloid leukemia (24.8\%) and acute lymphoblastic leukemia (29.5\%). From data of blood chemistry, typical physical symptoms of hematological malignancy undergoing chemotherapy such as anemia and nutritional deprivation were observed. ECOG performance status was Grade 1 (39.9\%), $2(31.2 \%)$ and $3(26.6 \%)$, which showed that physical activity of patients decreased in hospital to a varying extent. The mean hospitalization duration was $17.5 \pm 22.7$ days.

As indicated in Table 3, demographic and clinical variables were compared between the anxious/depressed group and the non-anxious/ depressed group among patients with hematological malignancy. There were no differences concerning age, BMI, blood chemistry, muscle strength, physical activity, ADL, or hospitalization period between groups. Concerning the EORTC QLQ-C30, global health status and emotion, cognitive, and social functioning significantly decreased in the anxious/depressed group compared with the non-anxious/
Table 1. Comparing psychological distress and muscle strength between patients with hematological malignancy and healthy controls

\begin{tabular}{|l|c|c|c|}
\hline & $\begin{array}{c}\text { Patients } \\
(\mathbf{n = 1 7 3 )}\end{array}$ & $\begin{array}{c}\text { Controls } \\
(\mathbf{n = 1 1 5})\end{array}$ & $\boldsymbol{p}$ \\
\hline Age (years) & $62.7 \pm 15.0$ & $66.0 \pm 13.5$ & .062 \\
\hline Sex & & & \\
\hline Male, n (\%) & $87(50.3)$ & $55(47.8)$ & .680 \\
\hline Female, n (\%) & $86(49.7)$ & $60(52.2)$ & \\
\hline Body mass index & $20.7 \pm 3.3$ & $22.7 \pm 2.9$ & $<.001$ \\
\hline HADS-anxiety & & & \\
\hline Mean & $6.3 \pm 3.7$ & $5.3 \pm 3.6$ & .031 \\
\hline$<8, \mathrm{n}(\%)$ & $116(67.1)$ & $83(72.2)$ & .357 \\
\hline$\leq 8, \mathrm{n}(\%)$ & $57(32.9)$ & $32(27.8)$ & \\
\hline HADS-depression & & & $<.001$ \\
\hline Mean & $7.4 \pm 3.7$ & $5.6 \pm 3.5$ & .009 \\
\hline$<8, \mathrm{n}(\%)$ & $94(54.3)$ & $80(69.6)$ & \\
\hline$\leq 8, \mathrm{n}(\%)$ & $79(45.7)$ & $35(30.4)$ & $<.001$ \\
\hline Handgrip, Kgf & $22.0 \pm 9.6$ & $30.4 \pm 10.2$ & .003 \\
\hline Knee extension strength, Kgf & $44.9 \pm 20.1$ & $52.1 \pm 19.2$ & \\
\hline
\end{tabular}

HADS: Hospital Anxiety and Depression Scale, Kgf: kilogram-force.

Table 2. Clinical characteristics among patients with hematological malignancy

\begin{tabular}{|l|c|}
\hline Clinical characteristics & \\
\hline Cancer type, $\mathrm{n}(\%)$ & $79(45.7)$ \\
\hline Lymphoma & $43(24.8)$ \\
\hline Acute myeloid leukemia & $51(29.5)$ \\
\hline Acute lymphoblastic leukemia & \\
\hline Blood chemistry & $9.8 \pm 2.0$ \\
\hline Hemoglobin, g/dL & $3.5 \pm 0.6$ \\
\hline Albumin, g/dL & $6.2 \pm 1.0$ \\
\hline Total protein, g/dL & $1.4 \pm 3.1$ \\
\hline C-reactive protein, mg/dL & \\
\hline ECOG performance status & $1.9 \pm 0.9$ \\
\hline Mean & $1(0.6)$ \\
\hline Grade 0, $\mathrm{n}(\%)$ & $69(39.9)$ \\
\hline Grade 1, $\mathrm{n}(\%)$ & $54(31.2)$ \\
\hline Grade 2, $\mathrm{n}(\%)$ & $46(26.6)$ \\
\hline Grade 3, $\mathrm{n}(\%)$ & $3(1.7)$ \\
\hline Grade 4, $\mathrm{n}(\%)$ & $17.5 \pm 22.7$ \\
\hline Hospitalization, days & \\
\hline Drug & $31(17.9)$ \\
\hline Opioid, $\mathrm{n}(\%)$ & $23(13.3)$ \\
\hline Sleeping drug, $\mathrm{n}(\%)$ & $6(3.5)$ \\
\hline Anti-anxiety, $\mathrm{n}(\%)$ & $5(2.9)$ \\
\hline Anti-depression, $\mathrm{n}(\%)$ & $3(1.7)$ \\
\hline Stabilizer, $\mathrm{n}(\%)$ & $27(15.6)$ \\
\hline Psychotropic drug, $\mathrm{n}(\%)$ & \\
\hline & \\
\hline
\end{tabular}

ECOG: The Eastern Cooperative Oncology Group.

depressed group. Concerning cancer-related symptoms, the anxious/ depressed group had more serious nausea, fatigue, insomnia, and appetite loss compared with the non-anxious/depressed group. Pain, dyspnea, constipation, and diarrhea did not differ between groups.

We used logistic regression analyses to investigate factors related to anxiety and depression. Emotion functioning (from the QLQ-C30) was excluded from independent variables intentionally because it predominantly assesses anxiety and depression $[25,26]$. The results revealed that age, nausea, and cognitive functioning were significantly associated with anxiety among patients (Table 4). Further, insomnia was significantly associated with depression (Table 5). All functions and physical symptoms were correlated anxiety (data not shown). 
Table 3. Differences between the anxious/depressed group and the non-anxious/depressed group among patients with hematological malignancy

\begin{tabular}{|c|c|c|c|c|c|c|}
\hline & Non-anxious & Anxious & & Non-depressed & Depressed & \\
\hline & $\mathrm{n}=116$ & $\mathrm{n}=57$ & $p$ & $\mathrm{n}=94$ & $\mathrm{n}=79$ & $p$ \\
\hline Age (years) & $63.7 \pm 14.9$ & $60.5 \pm 17.3$ & .207 & $62.4 \pm 16.7$ & $63.0 \pm 14.7$ & .798 \\
\hline Body mass index & $20.9 \pm 3.5$ & $20.4 \pm 2.8$ & .233 & $20.7 \pm 2.9$ & $20.6 \pm 3.7$ & .888 \\
\hline Hemoglobin, g/dL & $9.7 \pm 2.0$ & $10.1 \pm 1.9$ & .186 & $9.8 \pm 2.0$ & $9.8 \pm 2.0$ & .881 \\
\hline Albumin, g/dL & $3.5 \pm 0.6$ & $3.5 \pm 0.7$ & .884 & $3.5 \pm 0.6$ & $3.5 \pm 0.7$ & .982 \\
\hline C-reactive protein & $1.4 \pm 3.5$ & $1.3 \pm 2.0$ & .814 & $1.2 \pm 2.9$ & $1.5 \pm 3.3$ & .551 \\
\hline \multicolumn{7}{|l|}{ ECOG performance status } \\
\hline Mean & $1.9 \pm 0.9$ & $1.9 \pm 0.8$ & .189 & $1.9 \pm 0.9$ & $1.9 \pm 0.9$ & .631 \\
\hline Grade $0, \mathrm{n}(\%)$ & $1(0.9)$ & $0(0.0)$ & & $0(0.0)$ & $1(1.3)$ & \\
\hline Grade $1, \mathrm{n}(\%)$ & $50(43.1)$ & $19(33.3)$ & & $41(43.6)$ & $28(35.4)$ & \\
\hline Grade 2, n (\%) & $30(25.9)$ & $24(42.1)$ & & $28(29.8)$ & $26(32.9)$ & \\
\hline Grade 3, n (\%) & $32(27.6)$ & $14(24.6)$ & & $23(24.5)$ & $23(29.1)$ & \\
\hline Grade 4, n (\%) & $3(2.6)$ & $0(0.0)$ & & $2(2.1)$ & $1(1.3)$ & \\
\hline Handgrip, Kgf & $22.1 \pm 9.8$ & $21.6 \pm 9.3$ & .703 & $21.4 \pm 8.4$ & $22.6 \pm 8.4$ & .562 \\
\hline Knee extension strength, Kgf & $45.1 \pm 20.0$ & $44.5 \pm 20.7$ & .862 & $44.2 \pm 19.6$ & $45.6 \pm 20.9$ & .650 \\
\hline FIM & $117.5 \pm 14.1$ & $118.5 \pm 8.1$ & .630 & $117.8 \pm 12.6$ & $117.8 \pm 12.3$ & .983 \\
\hline Physical activity, daily steps & $1492.1 \pm 1074.5$ & $1625.6 \pm 1448.6$ & .635 & $1469.8 \pm 981.5$ & $1604.8 \pm 1404.3$ & .605 \\
\hline \multicolumn{7}{|l|}{ EORTC QLQ-C30 } \\
\hline Global health status & $53.9 \pm 21.5$ & $45.9 \pm 19.6$ & .020 & $54.2 \pm 22.2$ & $47.8 \pm 19.5$ & .049 \\
\hline Physical functioning & $69.4 \pm 18.3$ & $61.7 \pm 20.2$ & .133 & $68.7 \pm 19.4$ & $64.7 \pm 19.0$ & .174 \\
\hline Role function & $60.8 \pm 29.8$ & $49.7 \pm 33.4$ & .289 & $61.9 \pm 30.4$ & $51.5 \pm 31.8$ & .030 \\
\hline Emotion functioning & $79.5 \pm 15.7$ & $63.9 \pm 20.3$ & $<.001$ & $78.0 \pm 17.8$ & $70.0 \pm 19.1$ & .005 \\
\hline Cognitive functioning & $77.3 \pm 21.1$ & $65.2 \pm 24.9$ & $<.001$ & $77.7 \pm 23.0$ & $68.8 \pm 22.4$ & .013 \\
\hline Social functioning & $77.7 \pm 24.7$ & $60.8 \pm 31.1$ & $<.001$ & $76.6 \pm 28.6$ & $66.9 \pm 26.5$ & .023 \\
\hline Nausea & $6.3 \pm 11.6$ & $12.9 \pm 17.5$ & .004 & $8.5 \pm 13.6$ & $8.4 \pm 14.8$ & .974 \\
\hline Fatigue & $40.1 \pm 20.1$ & $47.4 \pm 22.1$ & .033 & $41.2 \pm 21.4$ & $44.0 \pm 20.5$ & .389 \\
\hline Dyspnea & $23.6 \pm 24.5$ & $28.1 \pm 23.4$ & .250 & $25.0 \pm 24.1$ & $22.3 \pm 22.1$ & .108 \\
\hline Pain & $24.9 \pm 23.3$ & $28.1 \pm 24.6$ & .404 & $25.9 \pm 23.7$ & $27.8 \pm 23.4$ & .328 \\
\hline Insomnia & $30.2 \pm 27.1$ & $43.9 \pm 33.4$ & .004 & $29.1 \pm 28.6$ & $41.4 \pm 30.3$ & .007 \\
\hline Appetite loss & $30.7 \pm 29.9$ & $40.3 \pm 29.4$ & .047 & $29.8 \pm 28.7$ & $38.8 \pm 30.9$ & .048 \\
\hline Constipation & $22.1 \pm 26.7$ & $24.6 \pm 23.2$ & .557 & $19.5 \pm 23.7$ & $27.0 \pm 27.3$ & .054 \\
\hline Diarrhea & $17.8 \pm 24.3$ & $22.8 \pm 3.6$ & .221 & $18.4 \pm 24.7$ & $20.7 \pm 25.8$ & .562 \\
\hline Financial impact & $21.0 \pm 28.3$ & $33.9 \pm 29.2$ & .006 & $21.3 \pm 28.0$ & $23.0 \pm 30.0$ & .051 \\
\hline Hospitalization, days & $17.1 \pm 23.1$ & $18.3 \pm 22.0$ & .741 & $18.8 \pm 23.5$ & $15.9 \pm 21.7$ & .418 \\
\hline
\end{tabular}

ECOG: The Eastern Cooperative Oncology Group, Kgf: kilogram-force, FIM: Function Independence Measure, EORTC: The European Organization for Research and Treatment of Cancer.

Table 4. Results from forward stepwise ordered logistic regression analyses regarding anxiety

\begin{tabular}{|c|c|c|c|c|}
\hline Factor & $\boldsymbol{\beta}$ & SE & OR (95\% CI) & p \\
\hline Age & -0.023 & 0.012 & $0.977(0.955,1.000)$ & .052 \\
\hline Nausea & 0.026 & 0.014 & .057 \\
\hline Cognitive functioning & -0.22 & 0.007 & .005 \\
\hline
\end{tabular}

SE: standard error, CI: confidence interval, OR: odds ratio.

Table 5. Results from forward stepwise ordered logistic regression analyses regarding depression

\begin{tabular}{|c|c|c|c|c|}
\hline Factor & $\boldsymbol{\beta}$ & SE & $\boldsymbol{p}$ \\
\hline Insomnia & 0.013 & 0.006 & .015 \\
\hline
\end{tabular}

SE: standard error, CI: confidence interval, OR: odds ratio.

\section{Discussion}

In the present study, the prevalence of depression was significantly higher among patients with hematological malignancy than healthy controls in Japan. However, the difference in the prevalence of anxiety between patients and healthy controls did not reach significance. The patients with hematological malignancy were more likely to suffer depression than healthy control, which might be a characteristic of patients with hematological malignancy.

We believed our results are meaningful because comparisons between patients with hematological malignancy and age-matched healthy controls are affected by various factors, including culture. In a French study, the mean HADS-anxiety and depression scores were $6.1 \pm 3.6$ and $3.8 \pm 3.4$ in healthy workers aged $>50$ years, respectively [27]. In Colombia, the mean HADS-anxiety and depression scores were $4.0 \pm 3.4$ and $4.7 \pm 3.9$ in healthy men aged $>60$ years, respectively [28]. One study showed severer depression among Japanese individuals compared to Americans [29]. Further, patients in the present study displayed more depression but less anxiety than did Brazilian patients with the same disease $(5.9 \pm 4.2$ and $7.9 \pm 4.7$, respectively) [17]. Therefore, depression and anxiety may be affected more by culture than disease-specific factors. 
Patients with anxiety had decreased global health status, emotion functioning, cognitive functioning, and society functioning, as well as increased nausea, fatigue, insomnia, appetite loss, and financial impact compared with non-anxious patients. Regarding the QLQ-C30, nearly identical results were reported in a previous study that examined Turkish patients with hematological malignancy [5].

Further, depression was also associated with physical functioning and physical symptoms among patients. However, several differences between depression and anxiety were observed. Concretely, nausea and fatigue were related to anxiety but not depression. The relationship between anxiety and nausea can be explained by classical conditioning: state anxiety directly facilitates the classical conditioning of nausea, and state anxiety exacerbates the magnitude of post-chemotherapy nausea experienced $[30,31]$. On the other hand, depressive symptoms and fatigue may share the same neuroendocrine-immune pathophysiologic mechanisms [32]. Therefore, a relationship between fatigue and depression was expected. However, in the present study, a relationship between fatigue and depression was not observed in patients with hematological malignancy, even though fatigue was associated with anxiety. Several prior studies also found no evidence for a causeand-effect relationship between depression and fatigue among cancer patients [33-35].

Significant relationships between anxiety and age, nausea, and cognitive functioning were detected in patients with hematological malignancy. Consistently, it was previously reported that anxiety increase with age in healthy people $[27,36,37]$. The relationship between nausea and anxiety is likely identical to that of nausea and depression (explained above). Further, cognitive functioning was previously affected by anxiety in a study examining older adults [38]. Moreover, a significant relationship between depression and insomnia was detected among patients with hematological malignancy. It is well known that insomnia and depression have a bidirectional relationship in patients without cancer $[39,40]$. Therefore, the fact that age, cognitive functioning, and insomnia were associated with anxiety/depression in the present study is not surprising. However, the relationship between anxiety and nausea may be critical for inpatients with hematological malignancy receiving chemotherapy, because anxiety can exacerbate the nausea felt during chemotherapy [30,31].

An unexpected result was that physical functioning and activity was not significantly related to either anxiety or depression in patients with hematological malignancy. There are many reports that showed a significant relationship between physical activity and psychological health in patients with/without cancer and healthy people [41-44]. However, in the present study, among healthy controls, physical functioning such as knee extension strength was related to depression (data not shown: $\mathrm{r}=-0.186, \mathrm{p}=.047,95 \% \mathrm{CI}:-0.357,-0.002$ ). It is also known that physical exercise can reduce psychological symptom such as anxiety/depression in patients with cancer $[45,46]$. In contrast, several studies showed that physical functioning was not associated with anxiety/depression in patients with cancer $[47,48]$; however, the number of these studies is negligible. The physical symptoms associated with chemotherapy might affect the relationship between physical function and psychological distress; therefore, further studies are necessary to elucidate this relationship.

Despite our large sample size, this study had some limitations. First, this study was conducted at a single-center. The observation research should be performed in multiple institutions. Second, several results could not be explained logically, even with referencing prior studies. The analysis of more detail factors should be added. Third, the cross-sectional design inhibits inferring causal relationships between variables. Prospective studies about anxiety and depression among patients with hematological malignancy should be conducted to support the development of effective interventions to manage their anxiety and depression.

In conclusion, the prevalence and severity of depression was higher than that of anxiety among Japanese inpatients with hematological malignancy, which differed from prior cross-cultural results. Regarding cancer-related physical symptoms in patients with hematological malignancy, nausea was greatly relevant to anxiety, and insomnia was associated with depression. On the other hand, physical functioning and activity were not related to either anxiety or depression in patients with hematological malignancy. Clinicians should focus on the mutual relationship between psychological distress and physical symptoms more. Early identification and proper intervention are therefore vital to minimize the negative effects of anxiety/depression on patients' QoL and the outcomes of their physical symptoms. Our findings can be used for both prevention and intervention among patients with hematological malignancy. Further studies are indispensable to discover potential pathophysiologic pathways between anxiety and depression in patients with hematological malignancy.

\section{Acknowledgments}

This research was supported by Japan Society for the Promotion of Science KAKENHI (grant number JP26282156 and 18K10711).

\section{References}

1. Knight K, Wade S, Balducci L (2004) Prevalence and outcomes of anemia in cancer: A systematic review of the literature. Am J Med 116: 11S-26S. [Crossref]

2. Kiernan J (2016) Genetic Influence on Chemotherapy-Induced Nausea and Vomiting: A Narrative Review. Oncol Nurs Forum 43: 389-393

3. Molassiotis A (2003) Anorexia and weight loss in long-term survivors of haematological malignancies. J Clin Nurs 12: 925-927. [Crossref]

4. Dimeo F, Schmittel A, Fietz T, Schwartz S, Kohler P, et al. (2004) Physical performance, depression, immune status and fatigue in patients with hematological malignancies after treatment. Ann Oncol 15: 1237-1242.

5. Pamuk GE, Harmandar F, Ermantas N, Harmandar O, Turgut B, et al. (2008) EORTC QLQ-C30 assessment in Turkish patients with hematological malignancies: association with anxiety and depression. Ann Hematol 87: 305-310.

6. Santos FR, Kozasa EH, Chauffaille Mde L, Colleoni GW, Leite JR (2006) Psychosocial adaptation and quality of life among Brazilian patients with different hematological malignancies. J Psychosom Res 60: 505-511.

7. Turhal NS, Dane F, Sinav H, Yalcin N, Khorshidi Z, et al. (2010) Anxiety and depression in Turkish breast cancer patients. $J$ BUON 15: 720-725. [Crossref]

8. Kang KD, Bae S, Kim HJ, Hwang IG, Kim SM, et al. (2017) The Relationship between Physical Activity Intensity and Mental Health Status in Patients with Breast Cancer. $J$ Korean Med Sci 32: 1345-1350. [Crossref]

9. Hagen KB, Aas T, Kvaloy JT, Eriksen HR, Soiland H, et al. (2019) Fatigue, anxiety and depression overrule the role of oncological treatment in predicting self-reported health complaints in women with breast cancer compared to healthy controls. Breast 28: 100-106.

10. Andrykowski MA, Steffens RF, Bush HM, Tucker TC (2014) Disparities in menta health outcomes among lung cancer survivors associated with ruralness of residence. Psychooncology 23: 428-436.

11. Oliveira Miranda D, Soares de Lima TA, Ribeiro Azevedo L, Feres O, Ribeiro da Rocha JJ, et al. (2014) Proinflammatory cytokines correlate with depression and anxiety in colorectal cancer patients. Biomed Res Int 2014: 739650. [Crossref]

12. Alacacioglu A, Ulger E, Varol U, Yavuzsen T, Akyol M, et al. (2014) Sexual satisfaction, anxiety, depression and quality of life in testicular cancer survivors. Med Oncol 31: 43 . [Crossref] 
13. Abuelgasim KA, Ahmed GY, Alqahtani JA, Alayed AM, Alaskar AS, et al. (2019) Depression and anxiety in patients with hematological malignancies, prevalence, and associated factors. Saudi Med J 37: 877-881.

14. Wang ZY, Liu L, Shi M, Wang L (2018) Exploring correlations between positive psychological resources and symptoms of psychological distress among hematological cancer patients: a cross-sectional study. Psychol Health Med 21: 571-582.

15. Clinton-McHarg T, Carey M, Sanson-Fisher R, Tzelepis F, Bryant J, et al. (2018) Anxiety and depression among haematological cancer patients attending treatment centres: prevalence and predictors. J Affect Disord 165: 176-181.

16. Priscilla D, Hamidin A, Azhar MZ, Noorjan KO, Salmiah MS, et al. (2018) Assessmen of depression and anxiety in haematological cancer patients and their relationship with quality of life. East Asian Arch Psychiatry 21: 108-114.

17. Bergerot CD, Clark KL, Nonino A, Waliany S, Buso MM, et al. (2013) Course of distress, anxiety, and depression in hematological cancer patients: Association between gender and grade of neoplasm. Palliat Support Care 13: 115-123

18. Miaskowski C, Wong ML, Cooper BA, Mastick J, Paul SM, et al. (2017) Distinct Physical Function Profiles in Older Adults Receiving Cancer Chemotherapy. J Pain Symptom Manage 54: 263-272. [Crossref]

19. Mystakidou K, Parpa E, Tsilika E, Panagiotou I, Zygogianni A, et al. (2012) Geriatric depression in advanced cancer patients: the effect of cognitive and physical functioning. Geriatr Gerontol Int 13: 281-288.

20. Vodermaier A, Millman RD (2011) Accuracy of the Hospital Anxiety and Depression Scale as a screening tool in cancer patients: a systematic review and meta-analysis. Support Care Cancer 19: 1899-1908.

21. Olssøn I, Mykletun A, Dahl AA (2005) The Hospital Anxiety and Depression Rating Scale: a cross-sectional study of psychometrics and case finding abilities in genera practice. BMC Psychiatry 5: 46. [Crossref]

22. Hashimoto R, Sakai A, Murayama M, Ochi A, Abe T, et al. (2015) Effects of dietary soy protein on skeletal muscle volume and strength in humans with various physical activities. J Med Invest 62: 177-183.

23. Kidd D, Stewart G, Baldry J, Johnson J, Rossiter D, et al. (1995) The Functional Independence Measure: a comparative validity and reliability study. Disabil Rehabil 17: $10-14$.

24. Aaronson NK, Ahmedzai S, Bergman B, Bullinger M, Cull A, et al. (1993) The European Organization for Research and Treatment of Cancer QLQ-C30: a qualityof-life instrument for use in international clinical trials in oncology. J Natl Cancer Inst 85: 365-376.

25. Tsunoda A, Nakao K, Hiratsuka K, Yasuda N, Shibusawa M, et al. (2005) Anxiety, depression and quality of life in colorectal cancer patients. Int J Clin Oncol 10: 411417.

26. Aminisani N, Nikbakht H, Asghari Jafarabadi M, Shamshirgaran SM (2017) Depression, anxiety, and health related quality of life among colorectal cancer survivors. J Gastrointest Oncol 8: 81-88. [Crossref]

27. Bocerean C, Dupret E (2014) A validation study of the Hospital Anxiety and Depression Scale (HADS) in a large sample of French employees. BMC Psychiatry 14: 354.

28. Hinz A, Finck C, Gomez Y, Daig I, Glaesmer H, et al. (2013) Anxiety and depression in the general population in Colombia: reference values of the Hospital Anxiety and Depression Scale (HADS). Soc Psychiatry Psychiatr Epidemiol 49: 41-49.

29. Matsumoto D, Nakagawa S, Estrada A (2009) The role of dispositional traits in accounting for country and ethnic group differences on adjustment. J Pers 77: 177-211. [Crossref]

30. Andrykowski MA (1990) The role of anxiety in the development of anticipatory nausea in cancer chemotherapy: a review and synthesis. Psychosom Med 52: 458-475.
31. Kamen C, Tejani MA, Chandwani K, Janelsins M, Peoples AR, et al. (2014) Anticipatory nausea and vomiting due to chemotherapy. Eur J Pharmacol 722: 172 179. [Crossref]

32. Lee BN, Dantzer R, Langley KE, Bennett GJ, Dougherty PM, et al. (2004) A cytokine-based neuroimmunologic mechanism of cancer-related symptoms. Neuroimmunomodulation 11: 279-292.

33. Byar KL, Berger AM, Bakken SL, Cetak MA (2006) Impact of adjuvant breast cancer chemotherapy on fatigue, other symptoms, and quality of life. Oncol Nurs Forum 33: E18-26. [Crossref]

34. Kim SH, Son BH, Hwang SY, Han W, Yang JH, et al. (2008) Fatigue and depression in disease-free breast cancer survivors: prevalence, correlates, and association with quality of life. J Pain Symptom Manage 35: 644-655.

35. Visser MR, Smets EM (1998) Fatigue, depression and quality of life in cancer patients: how are they related? Support Care Cancer 6: 101-108. [Crossref]

36. Laukka EJ, Dykiert D, Allerhand M, Starr JM, Deary IJ (2018) Effects of betweenperson differences and within-person changes in symptoms of anxiety and depression on older age cognitive performance. Psychol Med 48: 1350-1358. [Crossref]

37. Miloyan B, Byrne GJ, Pachana NA (2014) Age-related changes in generalized anxiety disorder symptoms. Int Psychogeriatr 26: 565-572.

38. Bierman EJ, Comijs HC, Jonker C, Beekman AT (2005) Effects of anxiety versus depression on cognition in later life. Am J Geriatr Psychiatry 13: 686-693. [Crossref]

39. Gebara MA, Siripong N, DiNapoli EA, Maree RD (2018) Effect of insomnia treatments on depression: A systematic review and meta-analysis. Depress Anxiety 35: 717-731. [Crossref]

40. Ohayon MM (2002) Epidemiology of insomnia: what we know and what we still need to learn. Sleep Med Rev 6: 97-111. [Crossref]

41. Branstrom R, Petersson LM, Saboonchi F, Wennman-Larsen A, Alexanderson K (2015) Physical activity following a breast cancer diagnosis: Implications for self-rated health and cancer-related symptoms. Eur J Oncol Nurs 19: 680-685.

42. Chipperfield K, Fletcher J, Millar J, Brooker J, Smith R, et al. (2013) Factors associated with adherence to physical activity guidelines in patients with prostate cancer. Psychooncology 22: 2478-2486.

43. Hiles SA, Lamers F, Milaneschi Y, Penninx BWJH (2017) Sit, step, sweat: longitudina associations between physical activity patterns, anxiety and depression. Psychol Med 47: 1466-1477. [Crossref]

44. Rebar AL, Stanton R, Geard D, Short C, Duncan MJ, et al. (2015) A meta-metaanalysis of the effect of physical activity on depression and anxiety in non-clinical adult populations. Health Psychol Rev 9: 366-378

45. Aguiñaga S, Ehlers DK, Cosman J, Severson J (2018) Effects of physical activity on psychological well-being outcomes in breast cancer survivors from prediagnosis to posttreatment survivorship. Psychooncology 27: 1987-1994. [Crossref]

46. Chen HM, Tsai CM, Wu YC, Lin KC, Lin CC (2014) Randomised controlled trial on the effectiveness of home-based walking exercise on anxiety, depression and cancerrelated symptoms in patients with lung cancer. Br J Cancer 112: 438-445

47. van Roekel EH, Bours MJ, Breedveld-Peters JJ, Willems PJ, Meijer K, et al. (2016) Modeling how substitution of sedentary behavior with standing or physical activity is associated with health-related quality of life in colorectal cancer survivors. Cancer Causes Control 27: 513-525. [Crossref]

48. Vallance JK, Boyle T, Courneya KS, Lynch BM (2015) Accelerometer-assessed physical activity and sedentary time among colon cancer survivors: associations with psychological health outcomes. J Cancer Surviv 9: 404-411.

Copyright: (C2019 Nakano J. This is an open-access article distributed under the terms of the Creative Commons Attribution License, which permits unrestricted use, distribution, and reproduction in any medium, provided the original author and source are credited. 